\title{
Branching of Sensory and Sympathetic Neurites in vitro Is Inhibited by Treatment with Taxol
}

\author{
Paul C. Letourneau, Terri A. Shattuck, and Alice H. Ressler \\ Department of Cell Biology and Neuroanatomy, University of Minnesota, Minneapolis, Minnesota 55455
}

Branching of elongating neurites in vitro occurs by the division of a growth cone into 2 or more daughter neurites. An important initial step is a broadening of the growth cone with establishment of a quiescent central growth cone margin. Within the spreading growth cone, microtubules and associated neuritic components diverge and become oriented toward the lateral protrusive margins of the leading edge (Letourneau, 1983). We have found that a low concentration of the microtubule-stabilizing agent taxol severely reduces the incidence of growth cone branching by cultured sensory and sympathetic neurons from chick embryos. In the presence of taxol, neurites are broader and have more microtubules than normally. Divergence of microtubules entering the growth cone from the proximal neurite is reduced in the presence of taxol, and quiescence of the central growth cone margin is less frequent. We propose that a critical step in branching is the separation and spreading of the neurite cytoskeleton by tensions generated at the lateral margins of the leading edge of the growth cone. Because taxol increases neurite size and microtubule content without increasing protrusive activity to the same extent, tensions produced in the motile leading edge are insufficient to spread the microtubules and associated neuritic materials into separate arrays for nascent branches.

Numerous studies have probed the roles of intrinsic and extrinsic factors in neuronal morphogenesis. Several in vitro studies have found that neurons generate axonal and dendritic shapes in culture that are distinctive and seem related to their in vivo morphologies (Banker and Cowan, 1978; Bray, 1973; Calvet et al., 1976; Kriegstein and Dichter, 1983; Neale et al., 1978; Wakshull et al., 1979). Because the in vitro environment is unlike the in vivo milieu in many ways, it is assumed that intrinsic factors have a role in this in vitro expression of recognizably characteristic neuronal shapes. Observations and manipulations in vivo also indicate that intrinsic factors determine important aspects of neuronal form (Sotelo and Arsenio-Nunes, 1976; Van der Loos, 1965).

It is clear, then, from a variety of studies that intrinsic regulatory factors contribute to the neuritic branching patterns of different neuronal types. In this paper we present evidence that the microtubular organization of elongating neurites is an important factor in the regulation of neuritic branching. Bray (1973) and Wessells and Nuttall (1978) have shown that most neurite branches arise in vitro by the division of a neurite tip into multiple growth cones, each leading a separate neurite. They described changes in growth cone shape and activity prior to and

Received Oct. 28, 1985; revised Jan. 3, 1986; accepted Jan. 6, 1986.

This research was supported by a grant from the Minnesota Medical Foundation, Grant PCM-8203855 from the National Science Foundation, and Grants HD 17192 and HD 19950 from the National Institutes of Health.

Correspondence should be addressed to Paul C. Letourneau, Department of Cell Biology and Neuroanatomy, 4-135 Jackson Hall, 321 Church St. S.E., University of Minnesota, Minneapolis, MN 55455.

Copyright (C) 1986 Society for Neuroscience $0270-6474 / 86 / 071912-06 \$ 02.00 / 0$ during branching that involve the filopodial and lamellipodial protrusions. In a previous study of the effects on in vitro neurite elongation of a broad range of concentrations of the drug taxol (Letourneau and Ressler, 1984), we found that a low concentration of taxol greatly reduces the frequency of neurite branching without greatly reducing the rate of elongation. In this further examination of neurite elongation in low taxol levels, we report that the cellular effects of this agent include an increase in neurite diameter and number of microtubules, decreased spreading of microtubules at the neurite tip, and a change in filopodial orientation at the growth cone. Perhaps molecules acting like taxol are intrinsic regulators of neurite branching and are distributed differently in neurons with different branching patterns.

\section{Materials and Methods}

\section{Cell culture}

Dorsal root ganglia (DRG) and sympathetic ganglia wcre removed from 9-11 d chick embryos, trypsin-dissociated, and cultured in an equal mixture of Ham's F12 (Gibco, Grand Island, NY) and heart conditioned medium (Luduena, 1973), supplemented with $10 \mathrm{ng} / \mathrm{ml}$ NGF (a gift from Donald Fink, University of Minnesota), as previously described (Letourneau, 1975). Medium, $1.5 \mathrm{ml}$, with approximately $5 \times 10^{4} \mathrm{DRG}$ or sympathetic neurons was plated into either (1) $35 \mathrm{~mm}$ tissue culture dishes (Falcon Plastics, Oxnard, CA) pretreated overnight with a 0.5 $\mathrm{mg} / \mathrm{ml}$ solution of poly(-1-ornithine) (Sigma Chemical Co., St. Louis, $\mathrm{MO}$ ) in borate buffer, or (2) $35 \mathrm{~mm}$ plastic petri dishes containing a 22 $\mathrm{mm}^{2}$ glass coverslip, carbon-coated, and pretreated with poly(-1-ornithine) (Letourneau, 1975). Some cultures were treated with $7 \times 10^{-9} \mathrm{M}$ taxol (Natural Products Branch, National Cancer Institute), dissolved in DMSO (Sigma Chemical Co., St. Louis, MO). Control cultures received the same amount of DMSO with no drugs. The cell cultures were incubated at $37^{\circ} \mathrm{C}$ in a $\mathrm{CO}_{2}$ incubator.

\section{Measurements of neurites and growth cones}

After $24 \mathrm{hr}$ the cultures were rinsed with warm PBS and fixed at $37^{\circ} \mathrm{C}$ with $1 \%$ glutaraldehyde in PBS for $30 \mathrm{~min}$. After rinsing in PBS and then water, the cells were stained for $1 \mathrm{hr}$ with a $5 \mathrm{mg} / \mathrm{ml}$ solution of tannic acid in water. Finally, the dishes were rinsed with water and stored at $4^{\circ} \mathrm{C}$. The cells plated on coverslips were mounted on slides in a solution containing polyvinyl alcohol.

Measurements of neurite length and branching of nerve fibers in tissue culture dishes were done using an Optomax image-analysis system attached to an Olympus inverted microscope. Neurites were viewed with a $20 \times$ objective, images were projected onto a video monitor, and neurite lengths were traced with a digitizing tablet while being viewed on the monitor. A neurite was defined as a cylindrical process at least as long as the diameter of a neuronal cell body, approximately $15 \mu \mathrm{m}$. This helped to eliminate confusion of neurites with filopodia, which average about $10 \mu \mathrm{m}$ in length (Letourneau, 1979). Measurements of growth cones were done on cells cultured on coverslips using a Zeiss IM microscope with a $63 \times$ planapochromat objective and phase-contrast optics. Images were projected onto a monitor with a Dage model 65 Newvicon video camera (Dage MTI, Inc., Michigan City, IN). Measurements or counts were made on the monitor with a ruler, and the distances were calibrated by projecting the image of a stage micrometer.

Several characteristics of growth cones were determined (Fig. 1). In 
making thesc mcasurements we samplcd growth cones from all areas of the coverslip but chose growth cones with configurations that permitted these measurements to be readily defined. Neurite width was measured proximal to the point of spreading at the base of the growth cone $\left(c-c^{\prime}\right)$. Growth cone spreading was measured as the maximum width of a growth cone from margin to margin but not including filopodia or lamellipodia $\left(b-b^{\prime}\right)$. Filopodial and lamellipodial extension was measured as the maximum distance between the distal tips of filopodia or lamellipodia at the opposite sides of a growth cone $\left(a-a^{\prime}\right)$. The angular distribution of filopodial orientation around the central axis of a growth cone was determined by placing a protractor on a central point at the base of a growth cone, aligned along the axis of the proximal neurite segment. The vectors of filopodial orientation to the left or right were then determined, as illustrated in Figure $1 d$, and scored in increments of $10^{\circ}$.

\section{Immunocytochemistry}

Cells on coverslips were rinsed with PHEM buffer (Schliwa and van Blerkom, 1981) and then simultaneously fixed and extracted for $20 \mathrm{~min}$ with $0.4 \%$ glutaraldehyde in PHEM buffer containing $0.2 \%$ Triton X-100 (Letourneau, 1983). The dishes were rinsed with $\mathrm{Ca} / \mathrm{Mg}$-free PBS and incubated $10 \mathrm{~min}$ in $\mathrm{Ca} / \mathrm{Mg}$-free PBS containing $1 \mathrm{mg} / \mathrm{ml}$ sodium borohydride. Before incubating cells with antibodies, the coverslips were soaked for $15 \mathrm{~min}$ in $\mathrm{Ca} / \mathrm{Mg}$-free PBS containing $5 \mathrm{mg} / \mathrm{ml}$ purified BSA (Miles Scientific, Naperville, IL) and $0.2 \%$ Triton X-100 (CMFPBS,TX,BSA). The coverslips were then covered with the primary antibodies, a mixture of 1:100 dilution of rabbit anti-chick smooth muscle actin (a generous gift of Dr. Judith Schollmeyer, Roman Hruska Meat Research Center; Letourneau, 1981) and 1:500 dilution of mouse monoclonal anti-chick $\beta$-tubulin (Amersham, Amersham, UK) in CMFPBS,TX,BSA, for $30 \mathrm{~min}$ at room temperature. The coverslips were rinsed and soaked again in CMFPBS,TX,BSA for $15 \mathrm{~min}$. The secondary antibodies were a mixture of 1:100 fluorescein-labeled goat anti-rabbit IgG (Cooperbiomedical, Inc., Malvern, PA) and 1:100 rhodamine-labeled goat anti-mouse IgG (Cooperbiomedical, Inc.) diluted in CMFPBS,TX,BSA and incubated on the coverslips for $30 \mathrm{~min}$ at room temperature. Finally, the coverslips were rinsed, soaked again for $15 \mathrm{~min}$ in CMFPBS,TX,BSA, rinsed again, and mounted in a polyvinyl alcohol solution on slides.

Cells were photographed on $35 \mathrm{~mm}$ film with the Zeiss IM microscope using a $63 \times$ planapochromat objective. Panatomic-X film was used for phase-contrast pictures and Tri-X Pan film for fluorescence microphotography.

\section{Electron microscopy}

Cells cultured in polyornithine-treated tissue culture dishes were fixed with glutaraldehyde, postfixed with osmium, embedded, sectioned, and staincd as described (Letourneau, 1983). The sections were observed and photographed with a Jeol 100CX EM.

\section{Results}

Except where noted, all these studies were done using neurons from DRG. Neurons cultured in the presence of $7 \times 10^{-9} \mathrm{M}$ taxol extend neurites that appear normal in many respects, except they are unbranched for long distances (Fig. 2, $a, b$ ). Mea-

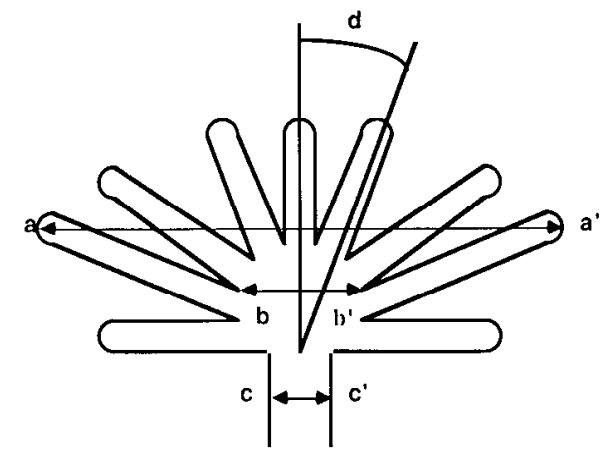

Figure 1. Schematic illustration indicating the locations of growth cone characteristics, as measured and reported in Table 1 and Figure 4. The positions of each measurement are defined in Materials and Methods.

surements of the mean length of neurite per neuron for sample populations of neurons with neurites and mean length of neurite per branch point are presented in Table 1. These data represent 3 different experiments and clearly show that the frequency of branch points is much reduced in the presence of taxol. In order to correct for the reduced mean length of neurite per neuron in taxol, we calculated branching as a function of neurite length, rather than branches per neuron. Because mean neurite width is nearly doubled by taxol (see below), the total mass of neurite produced is not reduced, but actually increased, by treatment with taxol. Despite the lower mean neurite length in the presence of taxol, some long neurites were formed, and unbranched neurites longer than $1000 \mu \mathrm{m}$ were measured in taxol-treated dishes. In order to examine the generality of this drug effect, we cultured chick sympathetic neurons in the same concentration of taxol, and found the same results as with the DRG neurons (Fig. 2, $c, d)$. The neurites of sympathetic neurons usually display much branching (Bray, 1973), but in the presence of taxol, neurites are unbranched or infrequently branched.

We examined characteristics of neurites and growth cones for differences that might be related to reduced branching in the presence of taxol. The mean neurite width in taxol was $1.9 \mu \mathrm{m}$ compared to $1.1 \mu \mathrm{m}$ in control dishes. If neurites have a cylindrical shape, then neurites are approximately 5.2 times more massive per unit length in the presence of taxol than in control medium. Because of the extreme difficulty of preparing ultrathin cross sections of individual cultured neurites from sparse, shortterm cultures, we did not determine the density of neuritic microtubules in control and taxol dishes. However, observation of longitudinal sections suggests that neurites in $7 \times 10^{-9} \mathrm{M}$ taxol contain as many or more microtubules per unit diameter as in control media (Figs. 6 and 7 in Letourneau and Ressler,

Table 1. Effects of taxol on neurite and growth cone characteristics

\begin{tabular}{lcc} 
Characteristic & Control $($ mean \pm SD) & Taxol $($ mean \pm SD) \\
\hline Neurite/neuron $(\mu \mathrm{m})$ & $503(96)$ & $287(104)$ \\
Neurite/branch point $(\mu \mathrm{m})$ & $248(96)$ & $981(104)$ \\
Neurite diameter, $c-c^{\prime}(\mu \mathrm{m})$ & $1.1 \pm 0.36(34)$ & $1.9 \pm 0.93(30)$ \\
Growth cone width, $\mathrm{b}_{-} \mathrm{b}^{\prime}(\mu \mathrm{m})$ & $8.3 \pm 7.34(34)$ & $7.6 \pm 4.65(30)$ \\
Filopodial spread, a- $\mathrm{a}^{\prime}(\mu \mathrm{m})$ & $28.8 \pm 12.7(34)$ & $28.2 \pm 7.6(30)$ \\
Filopodia/growth conc $(n)$ & $10.8 \pm 4.9(34)$ & $12.3 \pm 5.7(30)$
\end{tabular}

Neurons were cultured $\pm 7 \times 10^{-9} \mathrm{M}$ taxol, as described in Materials and Methods. The measurements of mean length of neurite per neuron and per branch point were made from duplicate dishes prepared in 3 different experiments. The measurements of growth cone characteristics (Fig. 1) were made from coverslips prepared in 2 experiments. Numbers in parentheses represent the number of neurons or growth cones measured. 
Figure 2. Inhibition of branching of sensory $(a, b)$ and sympathetic $(c, d)$ neurites by the presence of $7 \times 10^{-9}$ $\mathrm{M}$ taxol. Control neurites $(a, c)$ are frequently branched (arrows), while in the presence of taxol $(b, d)$ most neurites are unbranched. Note wider neurite diameter and clockwise bending (Letourneau and Shattuck, unpublished observations) commonly seen in taxol-treated cultures. $\times 325$.

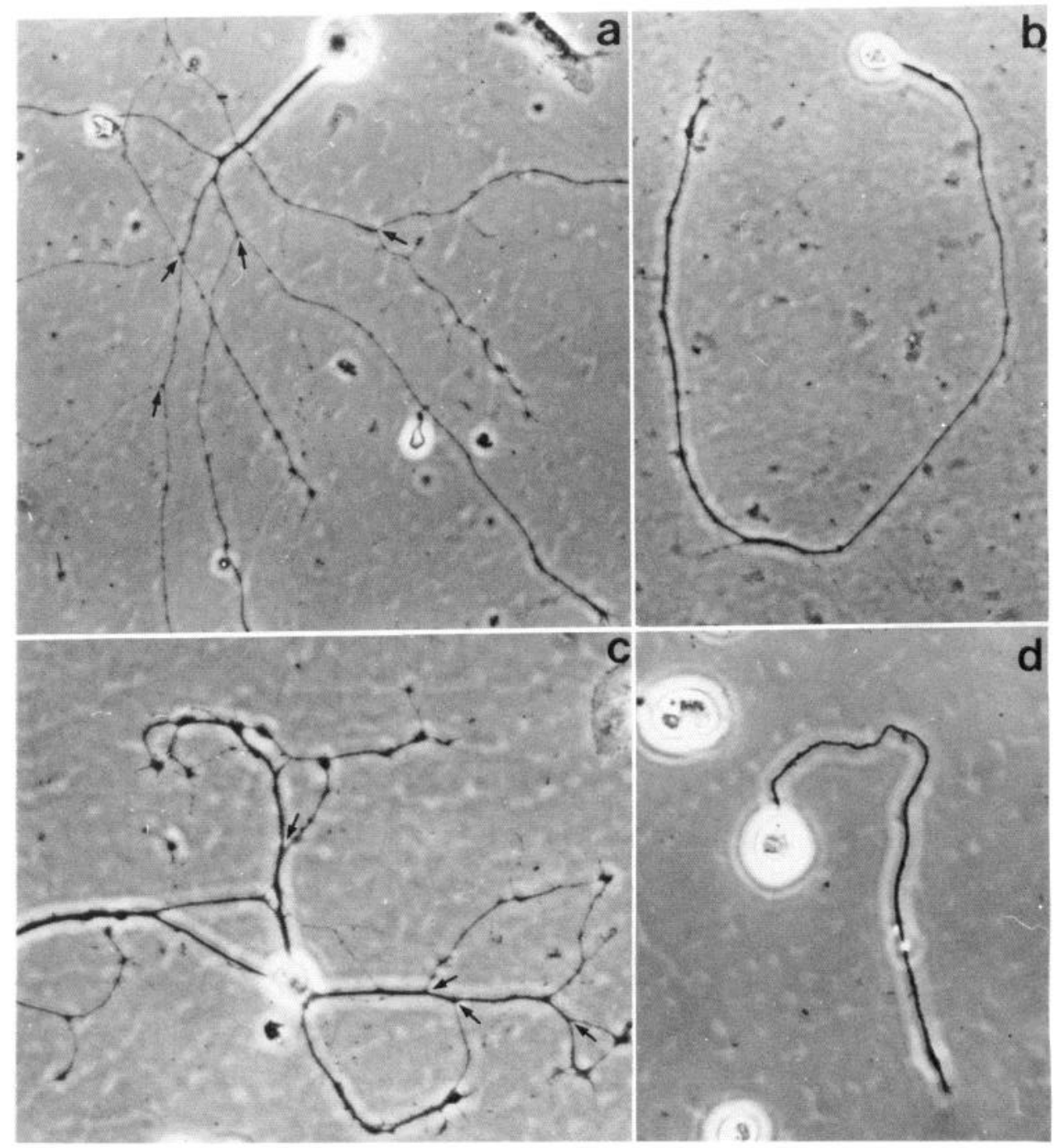

1984). This means that the total number of microtubules per neurite is substantially greater in the presence of taxol.

The relative distribution of actin filaments and microtubules in growth cones was examined in preparations double-stained for actin and tubulin (Fig. 3, $c-h$ ). This provided several clues to the relationship between microtubule organization and neurite branching. In control cultures, microtubules that extend from the neurite into the growth cone diverge from their compact arrangement in the neurite, spread apart, and terminate in the leading edge near the bases of filopodia and lamellipodia (Fig. 3, $c-f)$. In taxol, this splaying of microtubules at the neurite tip is less expansive (Fig. 3, $g, h$ ). Electron micrographs showing these features in extracted growth cones have been published previously (Letourneau, 1983; Letourneau and Ressler, 1984). Another characteristic feature of the distribution of microtubules was noted in regions of control growth cones that were not undergoing protrusive activity (i.e., absence of filopodia or lamellipodia). Very few microtubule ends project forward toward the cell margin at such sites, whether located along the sides of growth cones or at quiescent portions of the leading edge. Microtubules are diverted away from these areas, or, if present, they are oriented parallel to the cell margin (Fig. 3, $c-f$ ).

We found one difference among the motile characteristics of growth cones and filopodia that seems particularly related to reduced branching in the presence of taxol. Filopodia of taxoltreated growth cones are more likely to be oriented forward and are extended within a narrower range of angles than in control conditions (Figs. $3, a, b, g, h ; 4)$. In control cultures, $12 \%$ of the growth cone filopodia were oriented within $20^{\circ}$ of straight ahead and $28 \%$ were oriented away from the neurite axis by more than $80^{\circ}$. In the presence of taxol, $22 \%$ of filopodia were oriented within $20^{\circ}$ of straight ahead, more than the control cultures, and only $10 \%$ of filopodia diverged from the neurite axis by more than $80^{\circ}$ (Fig. 4). The 2 populations of filopodial angles shown in Figure 4 were compared using the Mann-Whitney $U$ test (Sokal and Rohlf, 1973), which indicated that the 2 samples are from significantly different populations at $p<0.001$. Wessells and Nuttall (1978) reported that the disappearance of filopodia from the central margin of the growth cone often precedes the division of a neurite tip into separate branches. Thus, growth cones in taxol tend to display filopodial orientation that is associated with an absence of branching activity.

Several other parameters of growth cones that are related to protrusive activity did not differ in the presence of taxol. The number of filopodia per growth cone was not significantly changed, despite the larger neurite diameter and volume in taxol medium. Also similar to the control conditions were the mean largest values for the width of growth cones and the lateral spread of filopodia (Table 1). 


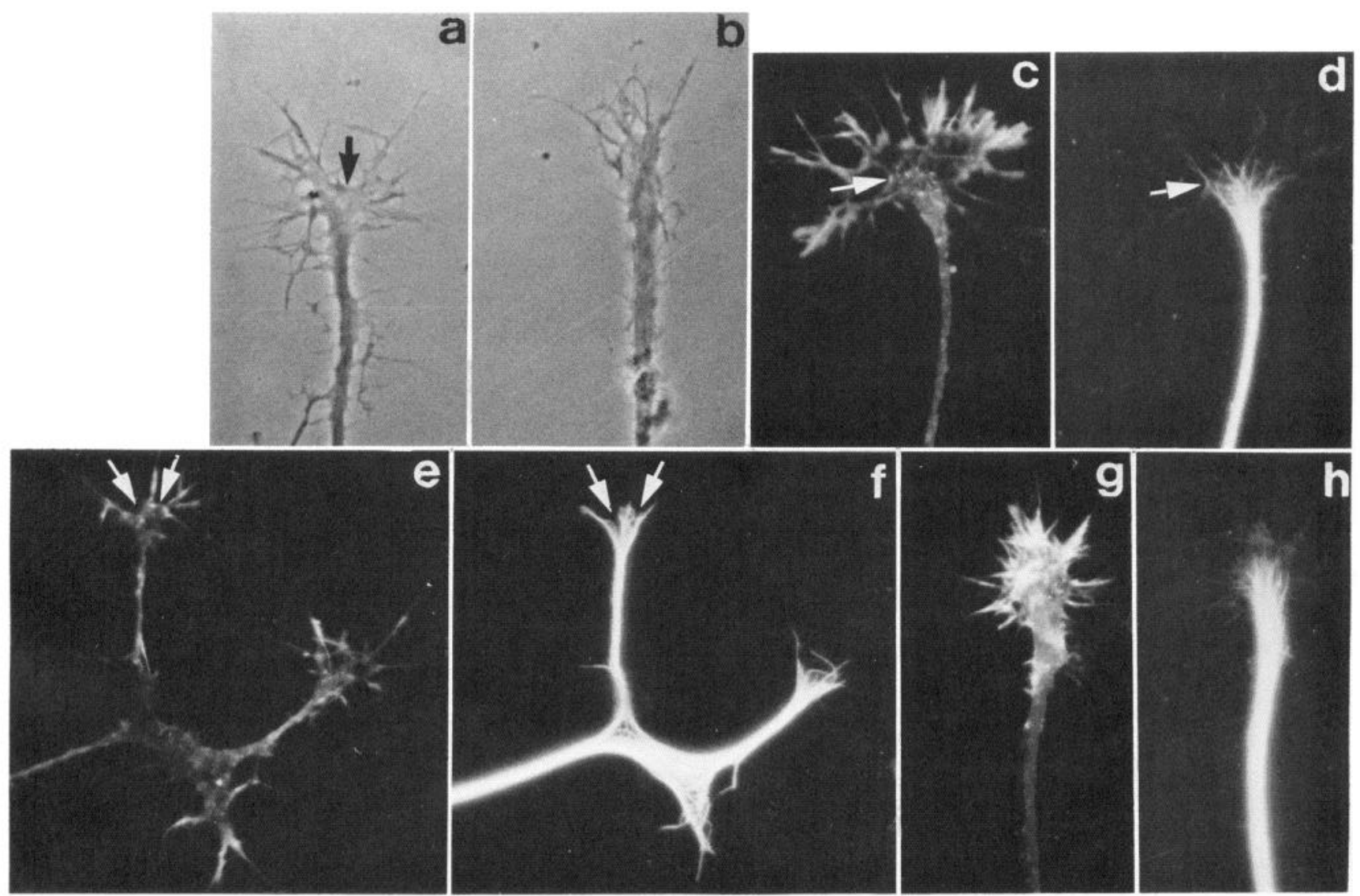

Figure 3. Phase-contrast micrographs of control $(a)$ and taxol-treated $(b)$ growth cones. Central quiescent area $(a r r o w$ in $a)$ often precedes branching of a growth cone. In $b$, note the wide neurite and forward orientation of filopodia induced by taxol. Double staining for actin $(c, e, g)$ and tubulin $(d, f, h)$ reveals relationships of microtubules and actin network in control $(c-f)$ and taxol-treated $(g, h)$ growth cones. Compare the greater divergence of microtubule ends in control growth cones $(d, f)$ vs a taxol-treated growth cone $(h)$. Note the orientation of microtubule ends away from nonmotile regions of the growth cone margin (arrows in $c-h) . \times 820$.

\section{Discussion}

We have found that the frequency of neurite branching is greatly reduced when neurites are cultured with a low concentration of taxol. Other effects of this treatment include increased neurite width and microtubule number, decreased spreading of microtubules at the neurite tip, and changes in filopodial orientation around the perimeter of the growth cone. Before offering an interpretation of these data, we review previous work on the mechanism of growth cone branching.

Bray (1973) observed sympathetic neurons in vitro and saw that nearly all branches arose by the bifurcation of a growth cone. He noted that branching involved initial broadening of the cone, leading to separate areas of lateral protrusion, and, finally, attenuation of the intermediate area into 2 daughter branches. Wessells and Nuttall (1978) analyzed autonomous branching and also induced branching by micromanipulation. They confirmed Bray's findings and emphasized that a critical initial event is broadening of a growth cone with active protrusion at the sides and less activity at the center. Along with this quiescence, the central region of the growth cone becomes detached or less adherent to the substratum, a process that may be accelerated by tensions generated at the adherent lateral margins. By using a microelectrode to release attachments at the central margin, Wessells and Nuttall could rapidly induce branching of most growth cones, and not only ones that were well spread. They concluded that the manipulations short-circuited the normal process by which stresses exerted from the

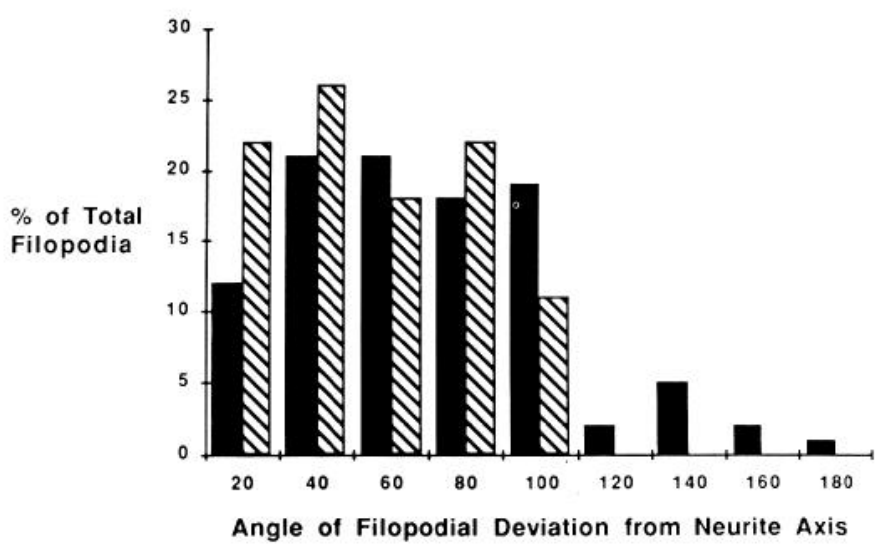

Figure 4. Histogram illustrating filopodial orientation away from the central neurite axis for growth cones from a sample of control and taxoltreated neurons. Measurements were made as described in Materials and Methods and Figure 1. The angles were plotted as degrees of deviation from $0^{\circ}$ (straight ahead) without regard to the side of the growth cone. A total of 108 filopodia were measured from control growth cones and 82 from taxol-treated growth cones. The solid black bars are filopodia of control growth cones, and the patterned bars are filopodia of taxol-treated growth cones. Note the narrower range of orientation in the presence of taxol, and the relatively fewer filopodia pointing forward on the control growth cones. 


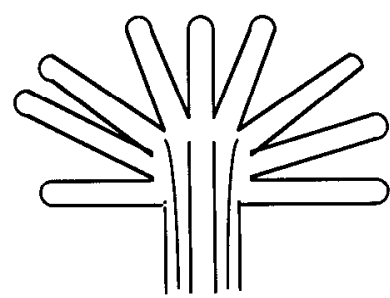

a
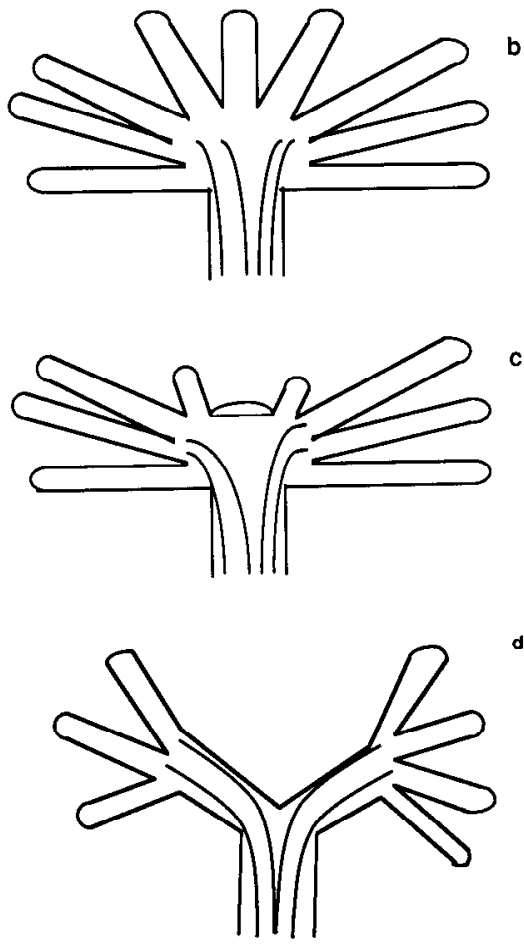

Figure 5. Model of growth cone branching, as described in the Discussion. The drawing depicts 4 microtubules within a growth cone with several filopodial projections. The microtubules begin to diverge as the growth cone broadens $(b)$. As the microtubules continue to be pulled laterally, the central margin becomes quiescent $(c)$, and as this process continues, 2 daughter neurites are resolved $(d)$.

lateral margins of a broadened growth cone induce or facilitate retraction of the central margin.

The interactions between actin filaments and microtubules are important in determining the shapes of elongating neurites (Joshi et al., 1985; Letourneau, 1979, 1983; Pollard et al., 1984). We have already observed at neurite tips that microtubules splay out from thcir compact alignment and extend individually or in small groups into the actin filament network at the leading margin (Letourneau, 1983). Potential contacts between microtubules and actin filaments are seen in extracted cytoskeletons, and though the associations between actin and microtubules in extracted cells may differ from the intact case, we have seen relationships in unextracted growth cones that also indicate interactions between these 2 cytoskeletal fibers (Letourneau, 1979). Perhaps, tensions exerted within the actin network are transmitted to these microtubules to promote their organization, polymerization, and, sometimes, separation into distinct cytoskeletal cores of daughter neurites. This influence on the distribution of microtubules at the leading edge of the growth cone may be crucial to neurite branching and turning.

The following is a hypothesis for neurite branching at the growth cone (Fig. 5). Assume that growth cone spreading is facilitated by substratum adhesion of filopodia at the sides of the growth cone. Growth cone spreading is much greater on substrata of high adhesivity (Letourneau, 1975, 1979). Mechanical forces produced in these attached lateral protrusions transmit tensions within the cytoskeletal network to induce the separation and lateral movement of microtubules and other components of the advancing neurite (Fig. $5 b$ ). This promotes the next crucial step, quiescence of the central growth cone margin. The shift in the cytoskeletal core of the neurite diverts the forward flow of cytoskeletal and membrane precursors away from the central margin and toward the lateral sites of tension (Fig. $5 c$ ). Lateral advance of the growth cone margin is thus enhanced at the expense of the central margin, which is slowed by the reduced supply of components. In support of this, we noticed that in control growth cones microtubules are not oriented toward portions of the growth cone margin that seem to be nonmotile (Fig. 3). As indicated by Wessells and Nuttall (1978), detachment of the central margin is critical in the resolution of daughter branches, and decreased motility at the central margin may lead to decay of its adhesive sites. Detachment may be further promoted by tensions exerted from the wellattached lateral motile edges. Eventually, the lateral movement of neuritic materials and the exertion of tensions on the central growth cone margins result in the resolution of 2 growth cones and daughter neurites (Fig. 5d). Thus, generation of tensions at lateral adhesive sites has 2 important roles in the branching process. One is lateral diversion of microtubules and other cytoskeletal components extending into the leading margin of the growth cone, and the other is force exerted within the cell surface to release cell-substratum adhesions at the less active central margin. As emphasized by Bray (1982), the tensions generated within filopodia have major roles in molding neuronal shape.

The simplest interpretation of our data is that taxol inhibits neurite branching by its stimulation and stabilization of microtubule polymerization. This leads to broad massive neurites with many microtubules. Our measurements of filopodial number and growth cone spreading indicate that the actin-bascd motile apparatus is not enlarged by taxol to the same degree as the neurite and its cytoskeletal core. Perhaps, a normal-sized actin filament system does not exert sufficient tensions to separate and spread the enlarged array of microtubules and associated cytoskeletal elements extending into the neurite tip. This fits with our observation that microtubules are not well spread in the growth cones of taxol-treated neurites. Besides affecting polymerization, taxol may have other effects on microtubules. In neurons treated with higher levels of taxol, microtubules are in closely packed arrays (Letourneau and Ressler, 1984), perhaps because of increased lateral associations between microtubules. If the low taxol levels used here also increase lateral associations between microtubules or with neurofilaments, this could also interfere with the diversion of microtubules by lateral tensions.

That taxol-treated growth cones tend to have more forwardly directed filopodia is consistent with our proposal. Even though growth cones spread to the same degree with or without taxol, the central region is less apt to become inactive in the presence of taxol. Perhaps, lateral tensions generated from the growth cone margin are less effective in promoting quiescence of the central zone, because they are less able to divert the larger microtubule core of taxol-treated neurites. This would permit the continued transport of precursors to the central margin. The narrower range of filopodial protrusion at the sides of a growth cone may also be related to the reduced lateral spread and flow of neuritic components in the presence of taxol. A recent analysis by Bray and Chapman (1985) found that filopodia are most commonly produced at the leading margin of growth cones and then move proximally to be resorbed or retracted at the more proximal lateral edges of the growth cone. A kinetic analysis such as theirs is needed to understand the changes in filopodia 
formation and removal brought about by this treatment with taxol.

In conclusion, low concentrations of taxol inhibit branching of neurites from DRG and sympathetic neurons in vitro. We propose that the larger neurite size and microtubule number induced by taxol decrease the ability of tensions exerted by lateral filopodia to spread the microtubules and associated neuritic components projecting forward from the base of the growth cone. Thus, central quiescence and detachment of the growth cone margin and subsequent resolution of separate neuritic shafts become unlikely. Intrinsic proteins may act like taxol to control the cytoskeletal organization of neurites. Some of the microtubule-associated proteins (Vallee et al., 1984) may have such activity. A recent paper reports abnormal axonal morphologies of identified neurons in mutants of the nematode $C$. elegans and suggests that the axonal cytoskeleton is altered in the mutant phenotype (Hedgecock et al., 1985). It would be interesting to compare these effects of taxol on growth cones and neurite characteristics with differences in the intrinsic characteristics of growth cones and neurites from neurons that express different branching patterns in vitro.

\section{References}

Banker, G., and W. M. Cowan (1978) Further observations on hippocampal neurons in dispersed cell culture. J. Comp. Neurol. 187: 469-494.

Bray, D. (1973) Branching patterns of individual sympathetic neurons in culture. J. Cell Biol. 56: 702-712.

Bray, D. (1982) Filopodial contraction and growth cone guidance. In Cell Behaviour, R. Bellairs, A. Curtis, and G. Dunn, eds., pp. 299318, Cambridge U. P., Cambridge, U.K.

Bray, D., and K. Chapman (1985) Analysis of filopodial movements of the neuronal growth cones. J. Neurosci. 5: 3204-3213.

Calvet, M. C., A. M. Lepault, and J. Calvet (1976) A procion yellow study of cultured Purkinje cells. Brain Res. 111: 399-406.

Hedgecock, E. M., J. G. Culotti, J. N. Thomson, and L. A. Perkins (1985) Axonal guidance mutants of Caenorhabditis elegans identified by filling sensory neurons with fluorescein dyes. Dev. Biol. 111: 158170.

Joshi, H. C., D. Chu, R. E. Buxbaum, and S. R. Heidemann (1985) Tension and compression in the cytoskeleton of PC 12 neurites. J. Cell Biol. 101: 697-705.
Kriegstein, A. R., and M. A. Dichter (1983) Morphological classification of rat cortical neurons in cell culture. J. Neurosci. 3: 16341647.

Letourneau, P. C. (1975) Possible roles for cell-to-substratum adhesion in neuronal morphogenesis. Dev. Biol. 44: 77-91.

Letourneau, P. C. (1979) Cell-substratum adhesion of neurite growth cones and its role in neurite elongation. Exp. Cell Res. 124: 127-138.

Letourneau, P. C. (1981) Immunocytochemical evidence for colocalization in neurite growth cones of actin and myosin and their relationship to cell-substratum adhesions. Dev. Biol. 85: 113-122.

Letourneau, P. C. (1983) Differences in the organization of actin in the growth cones compared with the neurites of cultured neurons from chick embryos. J. Cell Biol. 97: 963-973.

Letourneau, P. C., and A. H. Ressler (1984) Inhibition of neurite initiation and growth by taxol. J. Cell Biol. 98: 1355-1362.

Luduena, M. A. (1973) Nerve cell differentiation in vitro. Dev. Biol. 33: 268-284.

Neale, E. A., R. L. Macdonald, and P. G. Nelson (1978) Intracellular horseradish peroxidase injection for correlation of light and electron microscopic anatomy with synaptic physiology of cultured mouse spinal cord neurons. Brain Res. 152: 265-282.

Pollard, T. D., S. C. Seldon, and P. Maupin (1984) Intcraction of actin filaments with microtubules. J. Cell Biol. 99: 33s-37s.

Schliwa, M., and J. van Blerkom (1981) Structural interactions of cytoskeletal components. J. Cell Biol. 90: 222-235.

Sokal, R. R., and F. J. Rohlf (1973) Introduction to Biostatistics, Freeman, San Francisco.

Sotelo, C., and M. L. Arsenio-Nunes (1976) Development of Purkinje cells in absence of climbing fibers. Brain Res. 111: 389-395.

Vallee, R. B., G. S. Bloom, and W. E. Theurkauf (1984) Microtubuleassociated proteins: Subunits of the cytomatrix. J. Cell Biol. 99: 38s$46 \mathrm{~s}$.

Van der Ioos, H. (1965) The "improperly" oriented pyramidal cell in the cerebral cortex and its possible bearing on problems of neuronal growth and cell orientation. Bull. Johns Hopkins Hosp. 117: 228250.

Wakshull, E., M. I. Johnson, and H. Burton (1979) Postnatal rat sympathetic neurons in culture. I. A comparison with embryonic neurons. J. Physiol. (Lond.) 42: 1410-1425.

Wessells, N. K., and R. P. Nuttall (1978) Normal branching, induced branching and steering of cultured parasympathetic motor neurons. Exp. Cell Res. 115: 111-122. 